\title{
Modelización de costes en el transporte fluvial de mercancías. Aplicación al caso brasileño.
}

\author{
José Andrés Maroto Fernández \\ Dirección de Consultoría y Medio Ambiente, Ineco, España \\ Carlos Delgado Marón \\ Dirección de Consultoría y Medio Ambiente, Ineco, España
}

\section{RESUMEN}

El transporte fluvial de mercancías presenta ventajas comparativas respecto a otros modos debido a las importantes economías de escala que permite obtener para la movilización de grandes flujos en largas distancias. Su promoción resulta por lo tanto socialmente deseable en muchos contextos. Sin embargo, la correcta evaluación económica de actuaciones en este sistema de transportes (ya sean proyectos de mejora de las infraestructuras o de aplicación de políticas de transporte) requiere disponer de herramientas de análisis en profundidad de sus costes de producción.

El proyecto ha consistido en el desarrollo de un modelo de evaluación de costes para este modo de transporte en el que se consideran, entre otros, los efectos de las diferentes condiciones físicas de los canales de navegación y las características de las mercancías transportadas. La herramienta diseñada contempla una alta descomposición de los elementos de coste con el fin de permitir particularizar el cálculo a una amplia variedad de situaciones así como facilitar el análisis de sensibilidad respecto a distintos factores.

El modelo se estructuró en función de cuatro procesos que permiten la agrupación de centros de coste conceptual y funcionalmente homogéneos:

- Proceso canal: Donde se definieron los costes asociados a los canales de navegación (costes de capital, mantenimiento y gestión).

- Proceso esclusas: Evaluación de los costes de capital, mantenimiento y operación de las esclusas.

- Proceso transporte: Costes asociados a la operación de los servicios de transporte (empresas de navegación: capital, mantenimiento y operación).

- Proceso puertos: Costes de capital, mantenimiento y operación necesarios para el intercambio modal.

La estructura de los cálculos modelizados es homogénea para todos los procesos y su formulación sistemática. La herramienta desarrollada se ha aplicado a la estimación de los costes de producción del transporte fluvial en distintos ríos brasileños y para diferentes flujos de mercancías: gráneles sólidos, líquidos y tráfico ro-ro. 


\section{ENFOQUE CONCEPTUAL Y ESTRUCTURA DEL MODELO}

\subsection{Premisas metodológicas}

El objetivo central del trabajo consistió en el desarrollo de una metodología sistemática para la evaluación de los costes de transporte fluvial y su plasmación sobre un modelo de cálculo.

El modelo de evaluación desarrollado tiene como propósito fundamental estimar los costes unitarios para la definición de criterios de valoración de la eficiencia en la asignación de recursos por parte de la sociedad en el ámbito de la planificación de transportes. En ningún caso pretende predecir cifras reales sino modelizar una realidad compleja y analizar la influencia sobre los costes de diferentes escenarios de explotación.

El enfoque aplicado se basa en la estructuración en Procesos, lo que permite agrupar centros de costes homogéneos dependiendo de variables relacionadas, simplifica la identificación de variables y parámetros y posibilita simular un rango más amplio de posibles escenarios.

Se estructura en los procesos que caracterizan la cadena de valor del transporte fluvial: Proceso Puertos, Proceso Canal, Proceso Esclusa, Proceso Transporte.

\subsection{Proceso Puertos: partidas y centros de coste}

- Costes de Capital: Coste imputable a la disponibilidad y uso de las infraestructuras y equipamientos para la transferencia modal de la mercancía, su logística y almacenamiento representado por el valor de la depreciación del inmovilizado fijo. Los centros de coste más importantes son: Infraestructura viaria / ferroviaria de conexión; Canales de acceso; Infraestructuras de atraque, muelles y pantalanes; Infraestructuras de almacenamiento y Equipos de carga/descarga.

- Costes de Mantenimiento: Gasto monetario anual para asegurar las condiciones de funcionalidad de las infraestructuras y los equipamientos durante su vida útil.

- Costes de Operación: Coste de las actividades y suministros para la prestación de los servicios de trasbordo modal de la mercancía. Las partidas más relevantes son: personal, suministros, energía y combustibles.

\subsection{Proceso Canal: partidas y centros de coste}

- Costes de Capital: Coste aplicable al uso y disponibilidad de las infraestructuras y equipamientos del canal de navegación. Los centros de coste más importantes son: canales artificiales y obras de adaptación de los cauces naturales; áreas de reviro y maniobras para garantizar la seguridad y sistemas de soporte a la navegación.

- Costes de Mantenimiento: Asociados a las actividades necesarias para asegurar sus condiciones funcionales a lo largo de su vida útil.

- Costes de Operación: Costes de gestión y operación de las actividades y servicios 
proporcionados a las embarcaciones en el canal de navegación. Las partidas más relevantes son las correspondientes al personal de los centros de gestión.

\subsection{Proceso Esclusa: partidas y centros de coste}

- Costes de Capital: Atribuibles a la disponibilidad y uso de las infraestructuras y equipamientos en las esclusas. Los centros de coste relevantes son: Obras civiles (movimiento de tierras y estructuras) y compuertas e instalaciones de control.

- Costes de Mantenimiento: De conservación de funcionalidad durante su vida útil.

- Costes de Operación: Coste de los servicios prestados a las embarcaciones, fundamentalmente en las partidas de personal y energía.

\subsection{Proceso Transporte: partidas y centros de coste}

- Costes de Capital: Correspondientes a la disponibilidad y uso del inmovilizado para la prestación de los servicios de transporte fluvial (embarcaciones).

- Costes de Mantenimiento: Correctivo y preventivo de las embarcaciones.

- Costes de Operación: Asociados a la prestación del servicio de transporte. Las partidas más importantes son: Tripulación y personal; combustibles y lubricantes; seguros y gastos generales de la empresa de transporte.

\subsection{Estructura del modelo}

La estructura y el enfoque conceptual del modelo, se basa en las siguientes premisas:

- El modelo calcula el coste para un "cargamento tipo" (convoy completo), a partir de un conjunto de variables caracterizadoras y parámetros de operación.

- Identificación de los centros de coste relevantes en cada proceso, distinguiendo tres categorías: capital, mantenimiento y operación.

- El cálculo de cada elemento de coste para el cargamento analizado se efectúa siguiendo una estructura homogénea y mediante una formulación sistemática.

A partir de la información de base, (variables caracterizadoras y parámetros operacionales) los elementos conceptuales utilizados en el modelo para el cálculo de cada partida de coste, en los cuatro procesos (puertos, canal, esclusa y transporte) son:

- Coste Global (CG): importe anual de los gastos (para costes de operación y mantenimiento) o valor de depreciación del elemento productivo (para capital).

- Base de División (BD): productividad anual del activo, representada en las unidades apropiadas al correspondiente centro de coste.

- Producción de Transporte asociada a la carga (TF): valor del indicador de producción relativo al transporte de carga en cuestión, en las condiciones operacionales específicas, representado en las mismas unidades que la Base de División. 
De esta manera, la formulación genérica aplicada a cada partida del modelo es (1):

$$
\mathrm{CG} * \mathrm{TF} / \mathrm{BD}
$$

y por tanto la formulación para el coste del cargamento sería (2):

$$
\text { Coste Cargamento }=\Sigma(\mathrm{CG} * \mathrm{TF} / \mathrm{BD})
$$

Los cálculos para cada uno de los procesos se exponen en los siguientes epígrafes.

\section{CENTROS DE COSTE Y FORMULACIÓN}

\subsection{Proceso Transporte}

Las variables caracterizadoras y operacionales más relevantes, que alimentan el modelo y para las que se deben cargar valores conocidos, son las siguientes:

- Tamaño del cargamento (t) y Distancia del trayecto $(\mathrm{km})$,

- Productividad de los procesos de carga/descarga $(\mathrm{t} / \mathrm{h})$

- Velocidad del convoy fluvial y velocidad de la corriente del río o canal $(\mathrm{km} / \mathrm{h})$

- Esclusas en el trayecto y desmembramientos necesarios (un.)

- Coste de capital de las embarcaciones (M\$) y Potencia de los motores (HP)

- Remuneración mensual de las tripulaciones (\$/mes)

- Consumo de combustibles y lubricantes (litros/hora*HP) y Precios (\$/litro)

\begin{tabular}{|l|l|}
\hline \multicolumn{2}{|c|}{ COSTES DE CAPITAL DE LAS EMBARCACIONES } \\
\hline Coste Global & Valor de Capital - Valor Residual / Vida Económica \\
\hline Producción de Transporte & $\begin{array}{l}\text { Tiempo Total de Viaje }=\text { t. de carga + t. de navegación + } \\
\text { t. de esclusa + t. de desmembramientos + t. de espera } \\
\text { nocturno + t. de descarga + t. de retorno sin carga }\end{array}$ \\
\hline Base de División & Productividad anual: horas anuales de operación \\
\hline \multicolumn{2}{|c|}{ COSTES DE MANTENIMIENTO DE LAS EMBARCACIONES } \\
\hline Coste Global & Valor de Capital * Coeficiente de mantenimiento anual \\
\hline Producción de Transporte & Tiempo Total de Viaje \\
\hline Base de División & Productividad anual: horas anuales de operación \\
\hline \multicolumn{2}{|c|}{ COSTES DE LA TRIPULACIÓN } \\
\hline Coste Global & $\Sigma$ (No tripulantes * coste salarial medio) por categoría \\
\hline Producción de Transporte & Tiempo Total de Viaje \\
\hline Base de División & Productividad anual: horas anuales de operación \\
\hline \multicolumn{2}{|c|}{ COSTES DE COMBUSTIBLES Y LUBRICANTES (CÁLCULO DIRECTO) } \\
\hline Consumos & Consumo* Potencia * Factor de Utilización * Tiempo V. \\
\hline \multicolumn{2}{|c|}{ COSTES DE SEGUROS Y SUMINISTROS } \\
\hline Coste Global & $\begin{array}{l}\text { Seguro: valor capital embarcaciones * coeficiente prima } \\
\text { Suministros: importe anual estimado }\end{array}$ \\
\hline Producción de Transporte & Tiempo Total de Viaje \\
\hline Base de División & Productividad anual: horas anuales de operación \\
\hline
\end{tabular}

Tabla 1 - Resumen de los cálculos de la estimación de costes del proceso transportes 


\subsection{Proceso Esclusa}

Las variables caracterizadoras y operacionales adicionales que requiere el modelo para la evaluación de los costes del Proceso Esclusa son las siguientes:

- Coste de capital de las esclusas (M\$) y vida económica (años)

- Tráfico anual en cada esclusa (Número de esclusajes anuales)

- Número de empleados por categorías y remuneración $(\$ / m e s)$

- Remuneración del Personal por categorías (\$/mes)

\begin{tabular}{|l|l|}
\hline \multicolumn{2}{|c|}{ COSTES DE CAPITAL DE LAS ESCLUSAS } \\
\hline Coste Global & $\Sigma$ Valor de Capital - Valor Residual / Vida Económica \\
\hline Producción de Transporte & Número de esclusajes por las esclusas del trayecto \\
\hline Base de División & Tráfico anual en cada una de las esclusas (esclusajes año) \\
\hline \multicolumn{2}{|c|}{ COSTES DE MANTENIMIENTO DE LAS ESCLUSAS } \\
\hline Coste Global & Valor de Capital * Coeficiente de mantenimiento anual \\
\hline Producción de Transporte & Número de esclusajes necesarios para el paso del convoy \\
\hline Base de División & Tráfico anual en cada una de las esclusas \\
\hline \multicolumn{2}{|c|}{ COSTES DE OPERACIÓN DE LAS ESCLUSAS (PERSONAL) } \\
\hline Coste Global & Coste salarial anual de la plantilla \\
\hline Producción de Transporte & Número de esclusajes necesarios para el paso del convoy \\
\hline Base de División & Tráfico anual en cada una de las esclusas \\
\hline
\end{tabular}

Tabla 2 - Resumen del cálculo aplicado a la estimación de costes del proceso esclusas

\subsection{Proceso Canal}

La estimación de los costes asociados al proceso canal requiere además la asignación de valores al Valor de capital das infraestructuras e equipamientos (\$) y al Importe anual estimado de los gastos de mantenimiento y operación (\$)

\begin{tabular}{|l|l|}
\hline \multicolumn{2}{|c|}{ COSTES DE CAPITAL DEL CANAL } \\
\hline Coste Global & $\begin{array}{l}\text { (Valor de Capital - Valor Residual)* Distancia del } \\
\text { trayecto/ Vida Económica }\end{array}$ \\
\hline Producción de Transporte & Toneladas convoy analizado*Distancia del trayecto \\
\hline Base de División & Tráfico anual en el trayecto en toneladas*km \\
\hline \multicolumn{2}{|c|}{ COSTES DE MANTENIMIENTO DE LOS PUERTOS } \\
\hline Coste Global & Importe anual gastos de mantenimiento y operación \\
\hline Producción de Transporte & Toneladas transportadas en el convoy analizado \\
\hline Base de División & Tráfico anual en el trayecto en toneladas*km \\
\hline
\end{tabular}

Tabla 3 - Resumen del cálculo aplicado a la estimación de costes del proceso canal 


\subsection{Proceso Puertos}

El modelo requiere adicionalmente, para la estimación de los costes asociados al proceso portuario, la asignación de valores a las siguientes variables y parámetros:

- Tráfico anual en los puertos de embarque y desembarque (toneladas/año)

- Valor de capital de infraestructuras y equipamientos (\$) y vida económica (años)

- $\quad \mathrm{N}^{\mathrm{o}}$ de trabajadores por categorías y remuneración $(\$ / \mathrm{mes})$

- Suministros, seguros y otros gastos $(\$)$

\begin{tabular}{|l|l|}
\hline \multicolumn{2}{|c|}{ COSTES DE CAPITAL DE LOS PUERTOS } \\
\hline Coste Global & $\Sigma$ Valor de Capital - Valor Residual / Vida Económica \\
\hline Producción de Transporte & Toneladas transportadas en el convoy analizado \\
\hline Base de División & Tráfico anual en las instalaciones de origen y destino \\
\hline \multicolumn{2}{|c|}{ COSTES DE MANTENIMIENTO DE LOS PUERTOS } \\
\hline Coste Global & Valor de Capital * Coeficiente de mantenimiento anual \\
\hline Producción de Transporte & Toneladas transportadas en el convoy analizado \\
\hline Base de División & Tráfico anual en las instalaciones de origen y destino \\
\hline \multicolumn{2}{|c|}{ COSTES DE OPERACIÓN DEL PROCESO PORTUARIO (PERSONAL) } \\
\hline Coste Global & Coste salarial anual de la plantilla \\
\hline Producción de Transporte & Toneladas transportadas en el convoy analizado \\
\hline Base de División & Tráfico anual en las instalaciones de origen y destino \\
\hline \multicolumn{2}{|c|}{ COSTES DE SEGUROS Y SUMINISTROS } \\
\hline Coste Global & $\begin{array}{l}\text { Seguro: valor capital instalaciones * coeficiente prima } \\
\text { Suministros: importe anual estimado }\end{array}$ \\
\hline Producción de Transporte & Toneladas transportadas en el convoy analizado \\
\hline Base de División & Tráfico anual en las instalaciones de origen y destino \\
\hline
\end{tabular}

Tabla 4 - Resumen del cálculo aplicado a la estimación de costes del proceso puertos

\section{RESULTADOS: EVALUACIÓN DE COSTES Y ANÁLISIS DE SENSIBILIDAD}

La herramienta de simulación se aplicó a diferentes flujos de mercancías en los ríos brasileños (graneles sólidos agrícolas, líquidos y tráfico ro-ro) con los siguientes objetivos:

- Ajustar los valores de los parámetros de modelización en diferentes situaciones

- Análisis de la racionalidad y funcionalidad de la herramienta para la simulación de diversas situaciones y valoración de la eficiencia del sistema en diferentes escenarios

- La obtención de resultados concretos sobre los valores de coste unitarios que permitan la comparación con otros modos

Se presentan a continuación los resultados y conclusiones más relevantes obtenidos en el estudio de caso del transporte fluvial de graneles agrícolas en el río Madeira. 


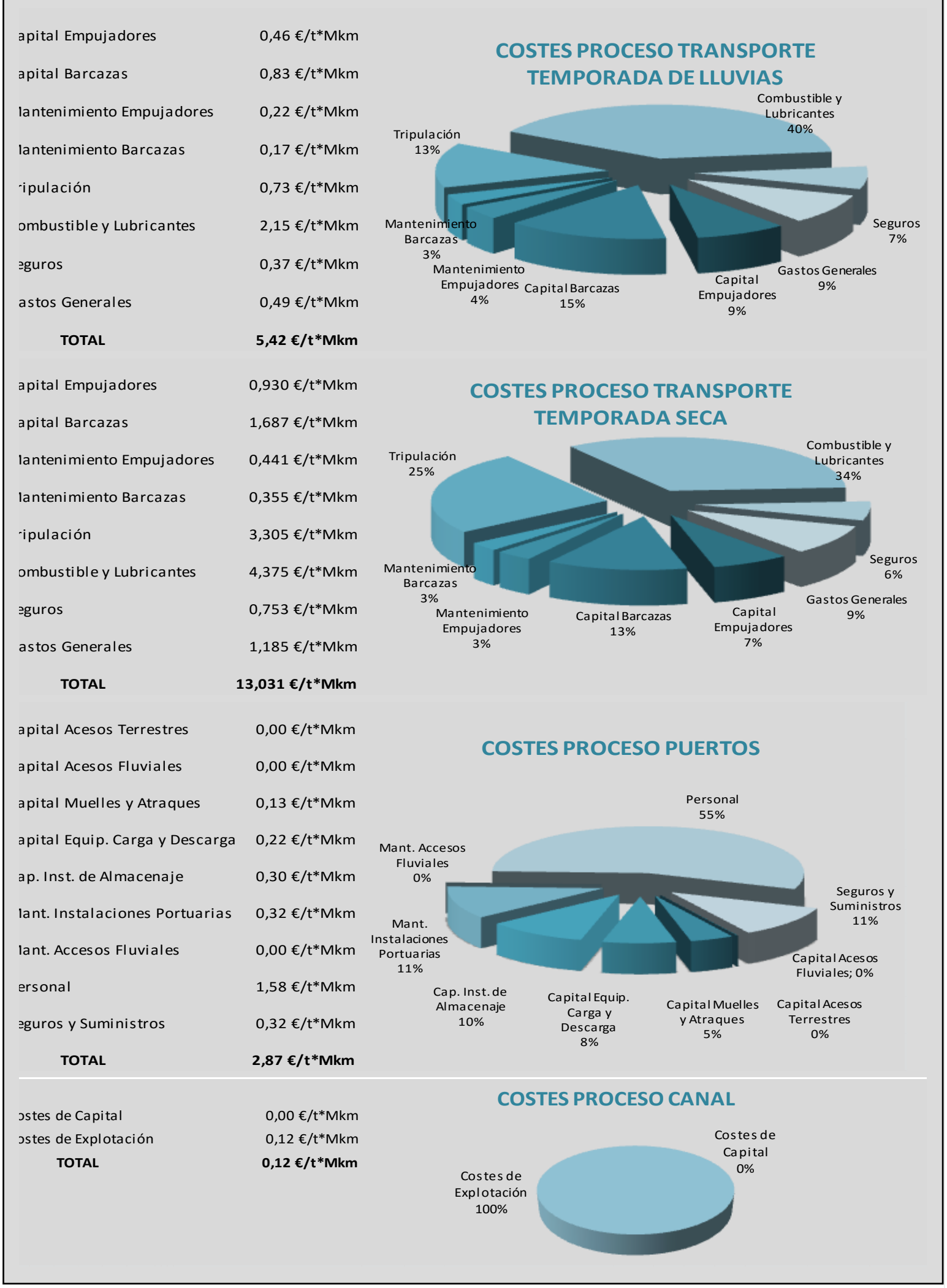

Fig. 1 - Resultados modelo estimación de costes. Evaluación de centros de coste en cada uno de los Procesos. Transporte de graneles sólidos en el río Madeira. 


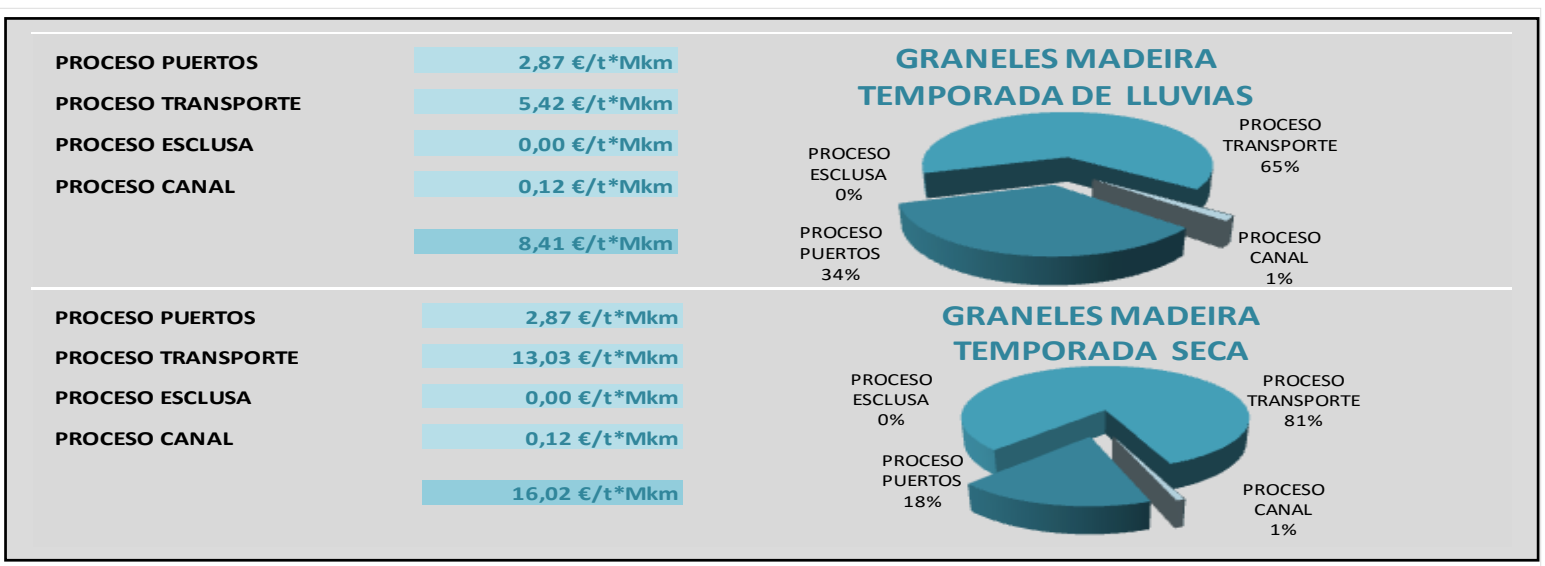

Fig. 2 - Resultados modelo estimación de costes. Costes totales y distribución por Proceso. Transporte de graneles sólidos en el río Madeira.
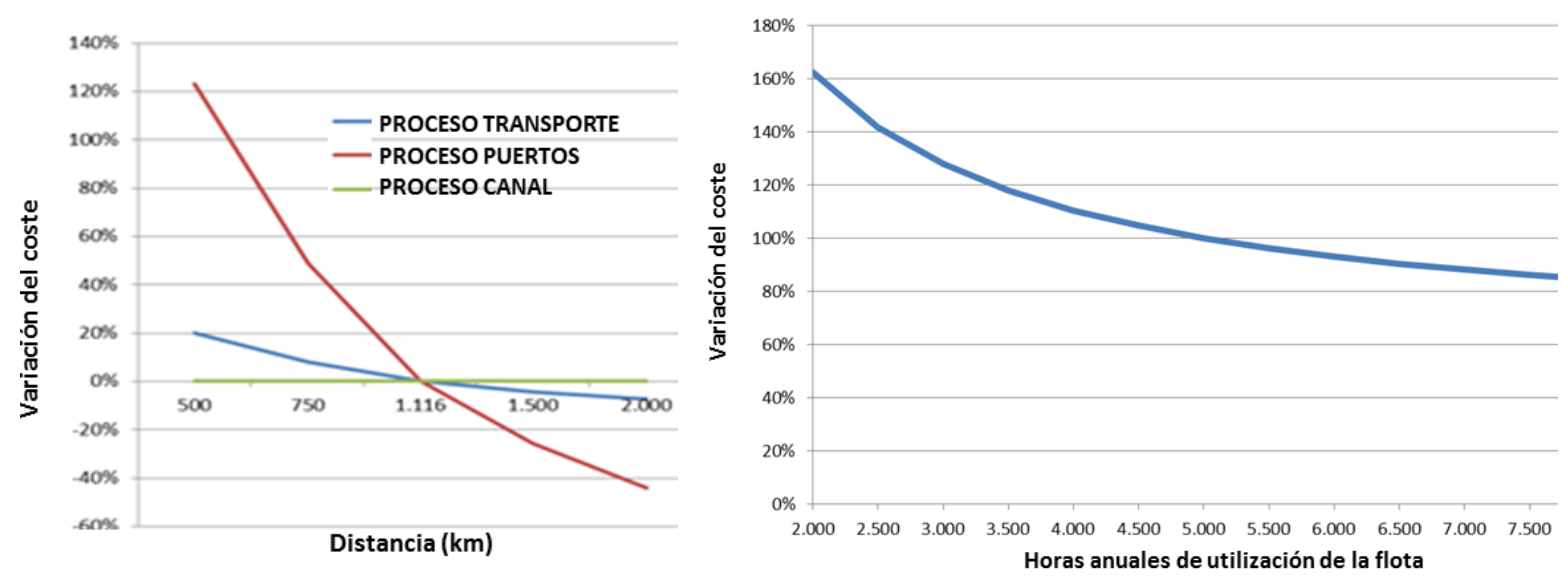

Fig. 3 - Sensibilidad del coste a la distancia y utilización de la flota.

Los principales resultados obtenidos son los siguientes:

- Los resultados presentan una elevada sensibilidad del coste entre temporada seca y de lluvias. Esto es consecuencia de las restricciones operativas sobre la dimensión de los convoyes (de $40.000 \mathrm{t}$ en temporada de lluvias y de $10.000 \mathrm{t}$ en temporada seca).

- El Proceso Transporte supone dos terceras partes del coste total en temporada de lluvias, y casi un $85 \%$ en temporada seca.

- Se detecta una elevada sensibilidad del coste a la hipótesis de utilización de la flota, aspecto muy vinculado al modelo de operación y gestión de los activos por parte del operador; por lo que este resultado debe manejarse con cautela en análisis comparativos.

- Los costes unitarios (por tonelada-Mil kilómetros) de los procesos Transporte y Portuario se sitúan por debajo de los 5,5€ y $3 €$ respectivamente. Los costes de ambos procesos se igualarían en el entorno de los $400 \mathrm{~km}$, con cifras próximas a 5,5€.

- Dada la magnitud hidrológica (caudal, calados y anchura) del río Madeira, los costes asociados al Proceso Hidroviario son marginales. Esta circunstancia hace innecesarias inversiones significativas en la hidrovía, limitando las posibilidades de conseguir eficiencias adicionales en el proceso logístico global. 